\title{
PAN-ARTHRODESIS FOR PARALYTIC FLAIL FOOT
}

\author{
Manuel Bastos Ansart, Barcelona, Spain
}

Paralysis of all the foot motors is rare as an isolated phenomenon, but it is common in conjunction with paralysis of other muscle groups in the lower limb. If one muscle remains active, it is usually the short flexor of the toes. The foot looks narrow, like a "midwife's hand." It is flaccid and, when bearing weight, is held at right angles to the leg, with a tendency to calcaneus; when not bearing weight, it hangs in equinus. There are always pronounced trophic and vaso-motor changes. The feet are cold and cyanosed, with chilblains in winter, especially on the toes and above the ankle on the posterior aspect of the leg. The scars of these may be visible. The victims are " slaves to their feet," which are a source of much misery, and may be found protected against pressure by adhesive strapping, cotton wool or bandages.

\section{INDICATIONS FOR OPERATION}

These flail feet undergo plastic changes as a result of the mechanical influences to which they are exposed. Treatment depends upon the static and dynamic state of the limb as a whole. If the foot can take weight without varus deformity no operation is needed. Usually, however, an operation is required in order to add strength and stability, or to correct deformity. The accepted procedure of fusion of the subtalar or midtarsal joints is theoretically sound, and controls the movements of pronation-supination or abduction-adduction, which are most likely to lead to severe deformity. In practice, however, no advantage is gained by the preservation of ankle movement. In fact, a mobile hind foot will predispose to late deformity. Moreover, if the ankle is fused, shortening of the limb can be compensated by fixing the foot in equinus. Thus the whole of the hind foot is converted into a solid unit leaving only the forefoot mobile. This is what happens when a normal woman wears high-heeled shoes.

In brief, the writer considers that the treatment of the flail foot with varus deformity and short leg should consist of fusion of the ankle, subtalar and midtarsal joints. The foot should be in equinus. The advantages claimed are: 1) good stability and prevention of late deformity; 2) compensation for shortening; and 3) ability to wear an ordinary high-heeled shoe, which is desirable for cosmetic and psychological reasons.

\section{PRINGIPLES AND TECHNIQUES OF OPERATION}

Complete fusion of the ankle and posterior part of the tarsus can be obtained by alternative methods: 1) implantation of an anterior bridge graft; or 2) peritalar intraarticular arthrodesis. The respective indications for either method are not definitely settled. In our practice the method adopted depends on the special circumstances of the case. When there is little tendency to valgus and the trophic changes are slight we prefer the anterior graft, particularly if the flail foot is complicated by complete instability of the knee. Fixed equinus of the foot, together with a plastic operation on the quadriceps muscle, completely changes the picture in these patients.

Peritalar intra-articular arthrodesis is indicated in the more severe cases of flail foot with poor circulation. The operation can also be used in cases of pronounced deformity, provided this can be corrected by a simple manipulation. We find that we have an increasing tendency to adopt peritalar intra-articular fusion, which is a much less severe operation than might be imagined. The results are uniformly satisfactory.

Anterior bridge graft: technique-A longitudinal incision is made from the mid-point of 


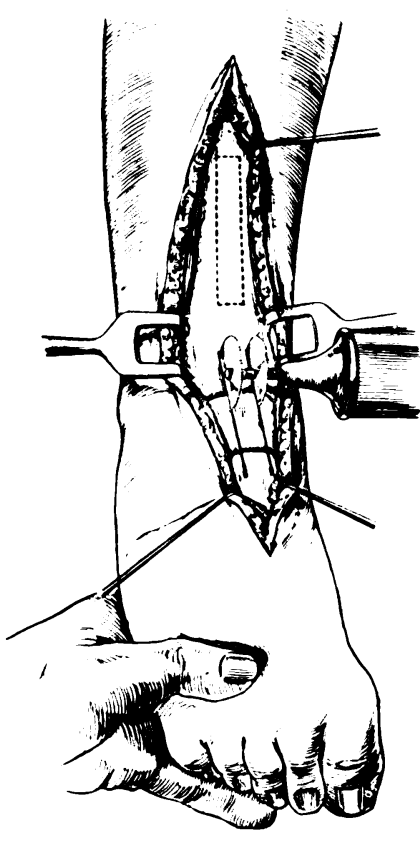

FIG. 1

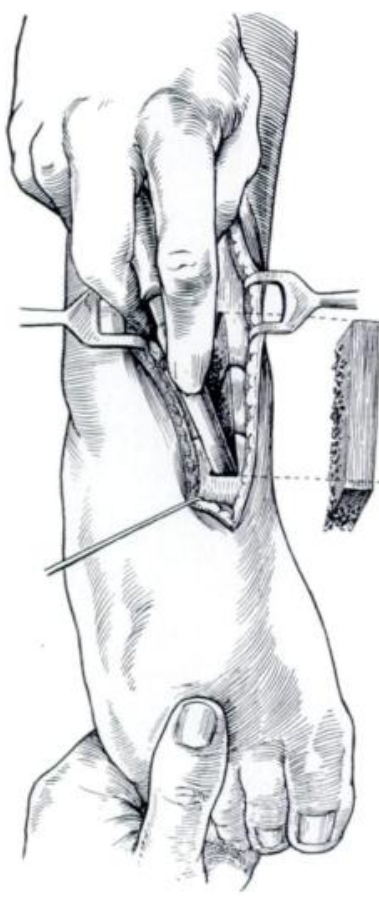

FIG. 2

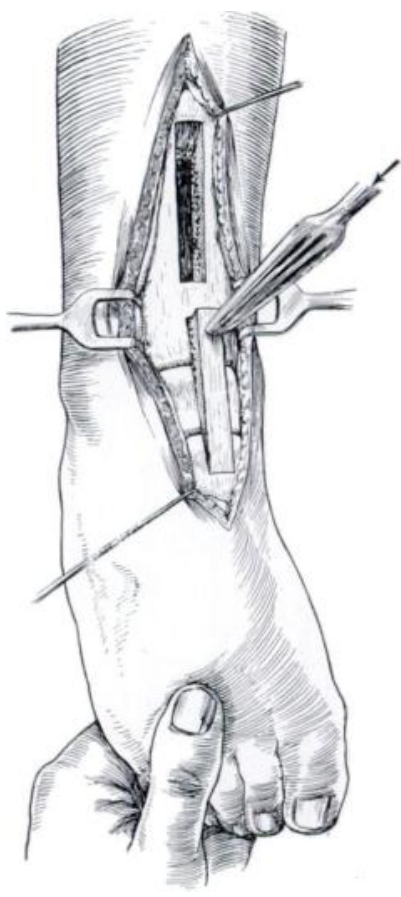

FIG. 3

Ankle and midtarsal arthrodesis by inlaid tibial graft. Figure $1-$ Cutting the slot for the graft. Interrupted lines indicate donor area. Figure 2-The slot is deepened with a gouge. Figure 3 -The graft inlaid.

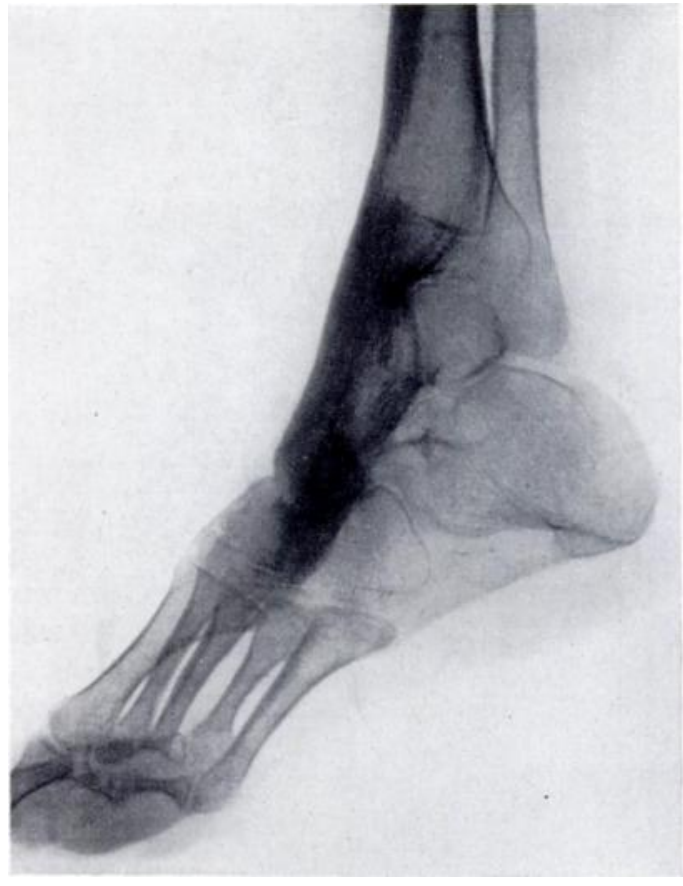

Fig. 4

Case 1-Paralytic flail foot. Successful fusion by double inlaid tibial grafts. Note position of equinus to compensate for limb shortening.

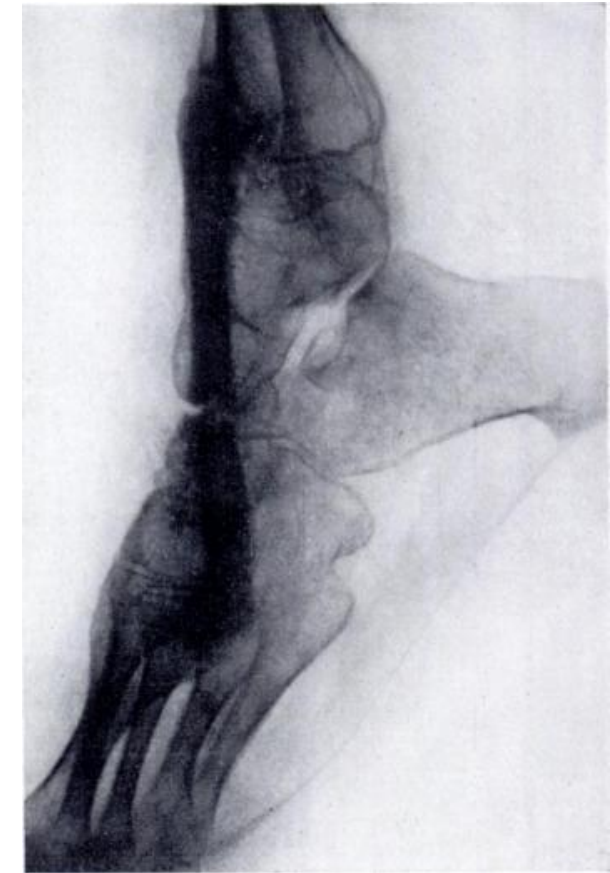

Fig. 5

Case 2-Anterior tibial graft for flail foot. The graft has broken near the lower end. Despite this, function of the foot was satisfactory. 
the shin to the dorsum of the foot. The incision is deepened, either medial or lateral to the extensor hallucis tendon and vascular bundle, according to the position chosen for the graft. The capsule of the ankle joint is opened and the periosteum is stripped from the tibia, talus and dorsal aspect of navicular. By means of a twin-bladed electric saw a deep ditch is made in a line from the anterior border of the tibia towards the first cuneiform; it should be about one centimetre wide and should extend from a point two centimetres above the articular surface of the tibia to the centre of the navicular (Figs. I and 2). A long graft is taken from the tibia and implanted firmly in the ditch (Fig. 3). The position of the graft should be chosen according to the side to which the foot is deviated: in a varus foot the graft should be placed medially, and in a valgus foot it should be more towards the lateral side. Sometimes two

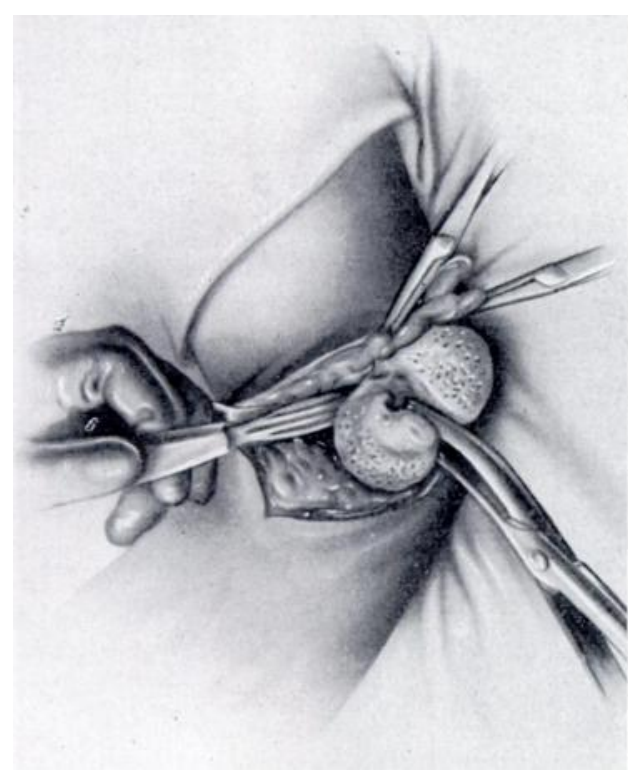

FIG. 6

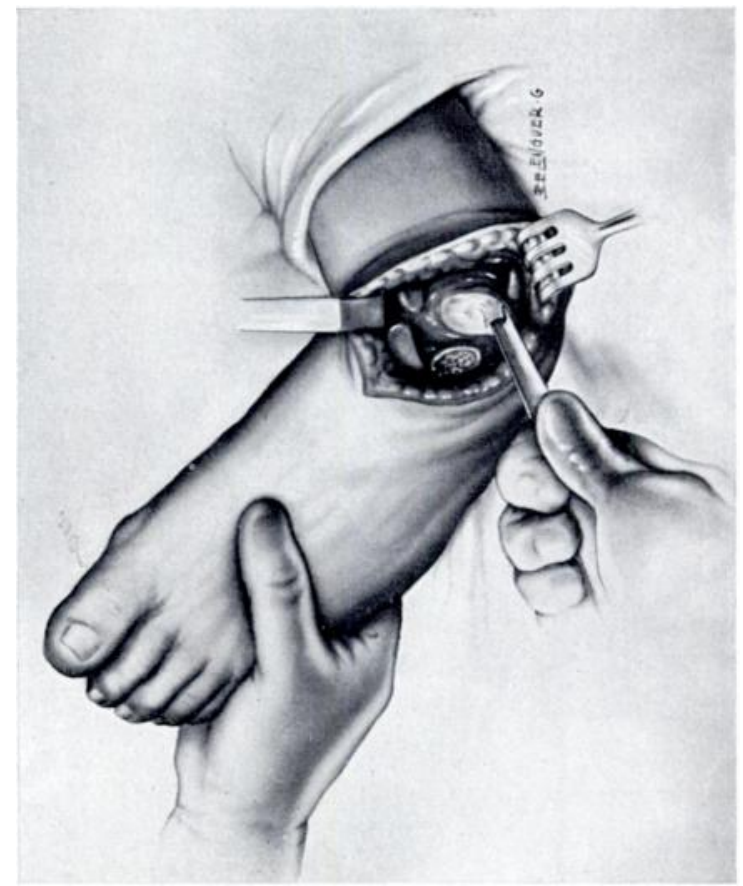

Fig. 7

Peritalar intra-articular arthrodesis. Figure 6-The talus is temporarily removed and denuded completely of articular cartilage. Figure 7-Before the talus is replaced the articular cartilage is stripped from the tibio-fibular mortise, from the calcaneum and from the navicular.

grafts are necessary, one medial, the other lateral. The graft should be a little longer than the ditch so that it can be sunk deeply into the spongy tissue of the medial cuneiform and, at the upper end, into the tibia. In order to do this, firm traction is applied to the foot, and when the graft has been inserted it is locked into position by releasing the traction. The periosteum and capsule of the ankle are sutured over the graft. The stitches should include the tendon and the lower part of the belly of the tibialis anterior. The periosteum of the donor area of the tibia is left open to permit free escape of blood. A posterior plaster shell is applied, and after ten days this is changed to a full length walking plaster which is retained for six months. At the end of this time the graft is usually found to be firmly fused (Fig. 4), but occasionally it may break or absorb at the level of the ankle joint (Fig. 5). This is not considered an operative failure because the remaining bony projections, one above, the other below, give fixity to the ankle. Sometimes the arthrodesis fails, and if some movement remains a painful pseudarthrosis may develop between the two ends of the broken graft. When this 


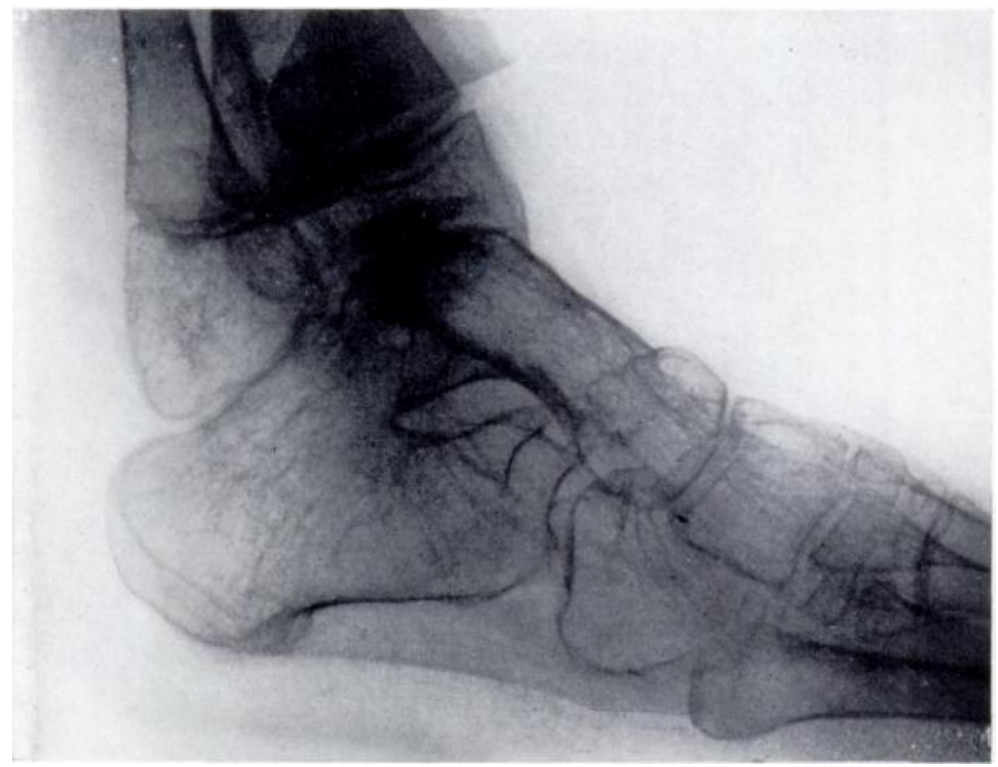

Fig. 8

Case 3-Peritalar intra-articular arthrodesis. Solid fusion one year after operation.

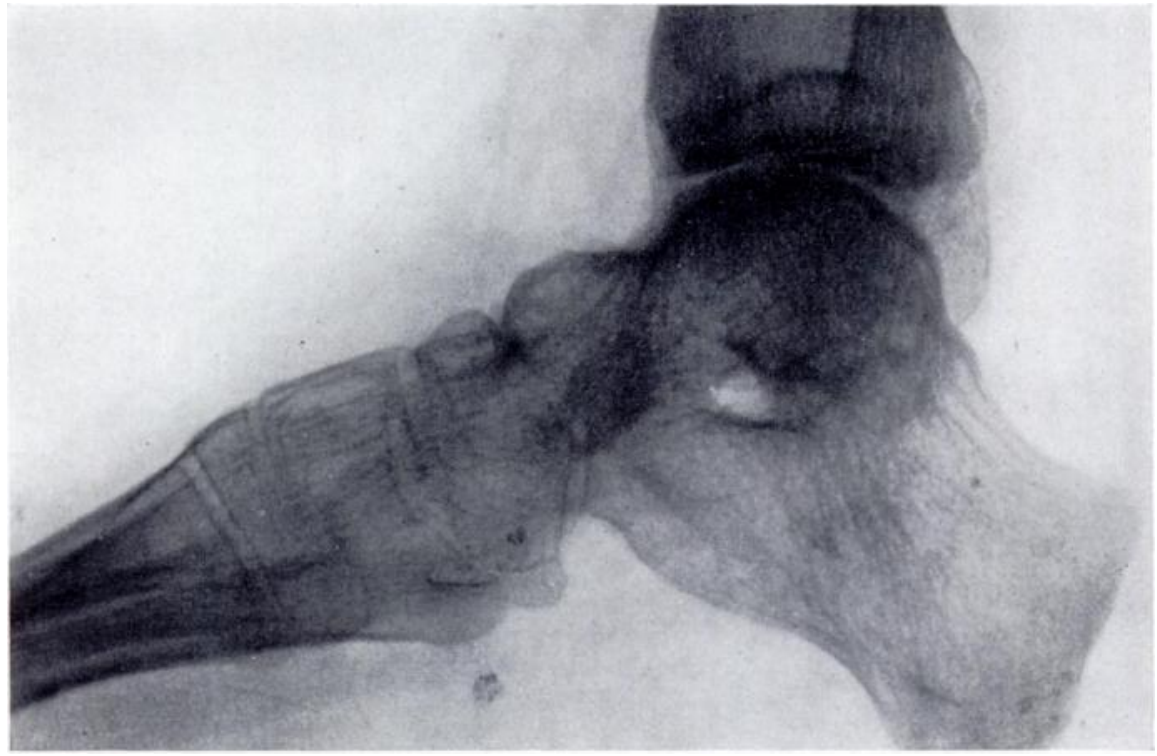

FIG. 9

Case 4-Another case one year after operation. There is firm consolidation of the talocalcaneal and talo-navicular joints, and bony trabeculae can also be seen crossing the ankle joint. 
happens, it is best to maintain plaster fixation for up to a year. The graft may then be found to be solid.

Peritalar intra-articular arthrodesis: technique-This operation is performed according to the technique of Lorthioir (1911) in which the talus is temporarily removed from its bed to allow thorough excision of all articular cartilage and exposure of cancellous bone. At first sight it seems somewhat venturesome to replace a part of the skeleton that has been removed from the body, but the fear of complications is ill-founded. We have never seen evidence of necrosis or tissue reaction after this procedure. On the contrary, fusion with the adjacent bone is rapid, and union may be seen in radiographs taken three or four months later. In these the talus appears comparatively normal in density, a fact which suggests that it is rapidly revascularised. The clinical course is likewise satisfactory.

An anterior incision is used. The talus must be removed without damage and without contact with the skin (Fig. 6). On the instrument table, the bone is freshened with a sharp wide chisel. Every fragment of cartilage must be removed and a thorough exposure made of cancellous bone. Removal of the talus allows free access to the surfaces of the tibio-fibular mortise, calcaneum and navicular, which are thoroughly denuded of cartilage (Fig. 7). To save time, the assistant should work on the talus while the surgeon prepares the bed, but if the two processes cannot be carried out together, the talus should be wrapped in a warm saline compress until it is replaced. The cavity is packed for a few moments before receiving the talus. The foot is then placed in the desired position and accurate apposition obtained by a few blows on the heel. In this way, good contact is obtained and dead spaces are eliminated. It is essential that perfect contact between the cancellous bone surfaces of the tibia, talus and calcaneum be obtained. A small horsehair drain is inserted and the capsule and skin sutured. Blood is expelled by compression and a few further blows applied to the heel to ensure contact. A posterior plaster slab is applied for ten days followed by an accurately fitting plaster which is retained for at least four months. At this time radiographs are taken, but it is sometimes necessary to maintain plaster fixation for a further period until fusion is complete.

The post-operative course is usually uneventful and the pain is slight. Patients are ready to walk early in plaster, and should be encouraged to resume normal life as soon as possible. Illustrative cases are shown in Figures 8 and 9.

\section{REFERENCE}

Lorthiolr, J. (1911): Quoted by Brandes (1934) in Verhandlungen der Deutschen Orthopädischen Gesellschaft. Kongress 28, p. 297. 